\title{
Sistema de recomendação baseado no ELO para problemas de pré-cálculo: Um experimento com calouros universitários
}

\author{
André Prisco, Rafael Penna, Michel Neves, Davi Teixeira e Silvia Botelho \\ ${ }^{1}$ Centro de Ciências Computacionais \\ Universidade Federal do Rio Grande (FURG) - Rio Grande - RS - Brazil \\ \{prisco.c3, daviltcomp, rapennas, nevess.michel, silviacb.botelho\}@gmail.com
}

\begin{abstract}
This work presents an experiment made with freshmen students from 3 university courses. They used a personalized system with pre-calculus problems. The objective was to evaluate how much our model and system can contribute to students' learning. We present the model and the results.
\end{abstract}

Resumo. Este trabalho apresenta um experimento feito com estudantes calouros de 3 cursos universitários. Eles utilizaram um sistema personalizado com problemas de pré-cálculo. O objetivo foi avaliar o quanto nosso modelo e sistema podem contribuir para o aprendizado dos estudantes. Apresentamos o modelo e os resultados.

\section{Introdução}

A personalização, abordagem importante em ambientes híbridos e em sala de aula, consiste em adaptar as atividades a serem desenvolvidas, levando em consideração o que o estudante está aprendendo, suas necessidades e habilidades, buscando colocar o estudante no centro do processo de ensino [Bacich et al. 2015]. O modelo em que todos os estudantes passam pelo mesmo processo ao mesmo tempo, produz estudantes desmotivados seja por não conseguir aprender no ritmo proposto enquanto outros, seja por terem de passar por atividades não desafiadoras para eles.

Os sistemas de recomendação educacionais surgem como ferramental para personalização mediando a escolha de objetos de aprendizagem e percebendo as necessidades educacionais do estudante.

Neste trabalho, apresentamos um sistema de recomendação educacional com o objetivo de apresentar desafios matemáticos personalizados no contexto do pré-cálculo. O sistema avalia o estudante conforme seu comportamento e busca entregar a ele um problema desafiador na medida certa. O objetivo é que o estudante encare desafios de diferentes categorias e mantenha-se motivado no processo.

Em trabalhos anteriores, propomos um modelo cognitivo para recomendação de objetos de aprendizagem. Avaliamos o modelo através do histórico de interação dos estudantes com uma plataforma de submissão de problemas de programação [Prisco et al. 2018, Prisco et al. 2017, Vargas et al. 2019, Zaffalon et al. 2019]. Neste trabalho desejamos avaliar o modelo em um contexto além da computação e em um ambiente de recomendação, no qual há de fato a intervenção ao estudante. Além disso, desejamos através deste protótipo oferecer um sistema que possa ser utilizado pela comunidade universitária (o que de fato está sendo feito por alguns professores). 
A interface do sistema é simples. O estudante recebe uma questão dada pelo sistema de recomendação, que ele acessa pelo seu computador ou dispositivo móvel. Ele tem acesso à descrição e ao material de apoio relacionado à questão. Também tem acesso às questões anteriores e ao material relacionado. A partir daí ele pode pular a questão ou submeter uma resposta. Caso ele submeta uma resposta, ele recebe um feedback informando se ele acertou ou não (o sistema não informou a resposta correta durante o período do experimento). Ao final da interação, o estudante pode dar o seu feedback acerca da questão e sua experiência na interação. Também pode informar o porquê de ter pulado. Após o seu feedback, o sistema gera nova recomendação que pode ser acessada pelo estudante.

O comportamento do estudante frente ao problema proposto serve como base da avaliação. A mediação é basicamente a decisão de qual a nova questão a ser recomendada. Avaliação e mediação são feitas pela integração de pressupostos da epistemologia genética aos modelos aplicados em jogos para escolha de jogadores, baseados na expectativa de desempenho na futura partida. Utilizamos o pré-cálculo como estudo de caso para este experimento. Além da relevância do tema para os calouros de exatas, os novos estudantes vêm com diferentes níveis de habilidade matemática, o que cria um bom ambiente para avaliar um sistema de personalização. Para escolha dos conteúdos, tivemos o apoio de dois especialistas, professores de cálculo da universidade. Eles formaram os diversos temas que deveriam ser abordados. A análise dos temas utilizou a BNCC, a matriz de referência do ENEM e o currículo de pré-calculo proposto no Khan Academy ${ }^{1}$ [BRASIL 2017, BRASIL 2009].

A universidade oferece um curso informal online para os estudantes. No entanto, a abordagem é padronizada e não atende o principal problema do estudante, que é a falta de habilidade algébrica e de resolução de problemas. O sistema proposto trabalha orientado a desafios, um caminho mais efetivo para o desenvolvimento de habilidade [Teixeira 2019, da SILVA 2011, Smole and Diniz 2009]. O centro da interação é a questão e é através dela que o estudante acessa o material de apoio disponível em formato de vídeos e textos. Espera-se que ele também pesquise outros materiais na Web.

Nas próximas seções, apresentamos o fundamento do nosso modelo, a arquitetura proposta e aplicada e os resultados obtidos.

\section{Reequilibração e expectativa de desempenho}

Nesta seção apresentamos alguns conceitos de técnicas de escolha de oponentes em jogos e da epistemologia genética e como integramos tais conceitos em nosso modelo de avaliação/mediação.

A epistemologia genética e os modelos pedagógicos relacionais compreendem a aprendizagem como a transformação tanto do sujeito (estudante) quanto do objeto a ser aprendido (em nosso caso, o objeto de aprendizagem e seu tema proposto) [Becker 2008, da SILVA 2011]. Como é na relação do objeto com o sujeito que ocorre a aprendizagem, fizemos um paralelo deste com os modelos aplicados em jogos, já que estes possuem técnicas para estudar as relações dos sujeitos-jogadores com os objetosadversários. Sistemas de matchmaking em jogos eletrônicos têm por objetivo encontrar

\footnotetext{
${ }^{1}$ https://pt.khanacademy.org/math/precalculus
} 
o melhor adversário para uma partida. Para um jogador que queira melhorar seu desempenho, ou seja, efetivar sua aprendizagem no jogo, as melhores partidas são aquelas em que ele pode melhorar sua técnica. Oponentes mais fracos são mais fáceis de vencer, mas oferecem pouca oportunidade de aprendizado, enquanto oponentes fortes demais podem ser desmotivadores ao jogador, que não tem o suporte para se apropriar de técnicas muito mais avançadas. Os sistemas então buscam encontrar adversários que potencializem o aprendizado. A mesma técnica pode ser adaptada para auxiliar na recomendação, não de adversários, mas de desafios na forma de objetos de aprendizagem.

A abordagem cognitiva na epistemologia genética aborda amplamente o desenvolvimento cognitivo tanto na infância quanto na vida adulta. Fundamentamos nosso trabalho no período pós-formal, que é o que estuda a aprendizagem de adultos [Knight and Sutton 2004]. Apresentamos alguns conceitos que utilizamos no decorrer deste trabalho, que são abordados em profundidade em [Piaget 1972, Lefrançois 2012].

O conhecimento são estruturas da mente que se relacionam a outras estruturas formando uma complexa rede. Tais estruturas, chamadas de esquemas, participam do processamento de informação, tomadas de decisões, processamento de padrões e etc. $\mathrm{O}$ sujeito está sempre interagindo com o meio, o qual chamamos de objetos. É na interação com os objetos que se dá a aprendizagem. A interação pode ser a leitura de um texto, dirigir um carro ou tentar resolver um problema de programação. Algumas vezes o objeto não fornece nenhum desafio para que o sujeito o assimile, este já possui os esquemas necessários para tal. Em outros casos, será necessário uma adaptação das estruturas mentais, para assimilar o novo objeto.

Dessa forma, a aprendizagem se dá pelo processo de adaptação do sujeito aos objetos aos quais interage. Quando o objeto não pode ser assimilado pela mente com as estruturas presentes, o sujeito entra em desequilíbrio. Neste caso há dois caminhos: desistir ou criar novos esquemas que preencham as lacunas. Essa construção e reconstrução de esquemas para assimilar novos problemas é chamada de acomodação. Denominamos reequilibração o processo do sujeito sair do seu equilíbrio em não conseguir assimilar o objeto e a construção de um novo equilíbrio, capaz de assimilar o objeto. Deve haver um balanceamento na reequilibração uma vez que o sujeito não se satisfaz somente assimilando os mesmos problemas aos quais já é capaz de interagir mas também tem a tendência de se desmotivar ao se deparar com problemas que necessitem de uma acomodação mais drástica aos esquemas já construídos [Piaget 1972].

Quando a mente se depara com séries de problemas apresentados de modo a respeitar a sua equilibração, esta pode se colocar em um estado de motivação cognitiva no aprender, ou seja, a própria curiosidade ou a inquietação por não assimilar o problema tornam-se motivadores de seu interesse na sua solução. O sujeito está em conflito cognitivo. Uma boa mediação, que promova a aprendizagem sob esta ótica, deve passar pela tarefa de oferecer ao sujeito objetos que potencialize uma balanceada reequilibração, apresentando caminhos que cooperem com este estado de motivação pela aprendizagem em si.

Os produtores de jogos possuem preocupação semelhante, e suas técnicas podem ser úteis. Em seus contextos, mediar é encontrar adversários que desequilibrem o sujeito, mas de forma cuidadosa de modo a desenvolvê-lo. O objetivo não é escolher o oponente 
que mais agrada, mas aquele que potencializa o ganho de habilidade e minimiza o risco de desmotivação.

Um dos modelos mais utilizados para esta tarefa foi desenvolvido para torneios de Xadrez e hoje é utilizado em diversos esportes e jogos eletrônicos. Os sistemas de classificação ELO encontram utilização em situações em que a habilidade de alguns "jogadores"deve ser estimada com base em resultados de competição entre pares. O modelo matemático e uma avaliação mais detalhada do nosso modelo é descrito em como descrito em [Langville and Meyer 2012, Elo 1978, Prisco et al. 2017, Prisco et al. 2018, Zaffalon et al. 2019].

Neste trabalho, apresentamos como hipótese de que o $\Delta$ entre o ELO do sujeito e o ELO do objeto pode ser usado para inferir o salto de desenvolvimento que o estudante deverá ter para assimilar o objeto de aprendizagem.

Ao observarmos uma partida como uma relação sujeito-objeto e a construção de um matchmaking como uma mediação pedagógica, podemos então fazer o caminho inverso e utilizar a expectativa de desempenho como ferramenta pedagógica. Sob esta abordagem, a expectativa de desempenho modelada em nosso trabalho pelo ELO rating é usada para avaliar o potencial de reequilibração que a relação sujeito-objeto oferece e a mediação é recomendar objetos que melhor se adequem ao sujeito, potencializando o conflito cognitivo [Piaget 1972, da SILVA 2011].

Uma boa mediação, sob este aspecto, consiste em priorizar a interação com objetos de aprendizagem que potencializem a reequilibração. Acreditamos que interações cujo $\Delta$ seja positivo mas pequeno (propiciando um desafio exequível, com incerteza sobre o resultado), são as que mais potencializarão ao estudante a experiência da reequilibração e do conflito cognitivo.

\section{Uma arquitetura para Sistema de Recomendação basada em Expectativa de Desempenho à luz da Epistemologia Genética}

A Figura 1 apresenta a arquitetura de nosso modelo. Os dados sobre os estudantes e objetos de aprendizagem são armazenados nos módulos de representação. O módulo de mediação utilizam estas informações para tomada de decisão e o módulo de avaliação atualiza as representações. Os módulos são descritos a seguir.

O estudante é o centro da arquitetura e representa o sujeito em seu processo de aprendizagem. Os objetos de aprendizagem adotados são questões objetivas a fim de facilitar a correção automatizada. Cada estudante e cada objeto possui um rating que é o seu valor de ELO. A cada nova interação, os valores de ELO são atualizados. A Figura 2 apresenta a representação de um estudante em nosso modelo.

\subsection{Avaliação}

Representamos a avaliação como se o resultado da interação fosse o final de uma partida entre o estudante e o objeto de aprendizagem. Usamos a metáfora do objeto de aprendizagem desafiando o estudante. Se o desfecho da interação foi bem sucedido, podemos dizer que o estudante venceu o duelo, e que o objeto de aprendizagem perdeu. Se o desfecho não foi bem sucedido, podemos dizer que o objeto de aprendizagem venceu. Em caso de o estudante pular a questão, deixamos os valores de ELO inalterados. Dessa forma, o 


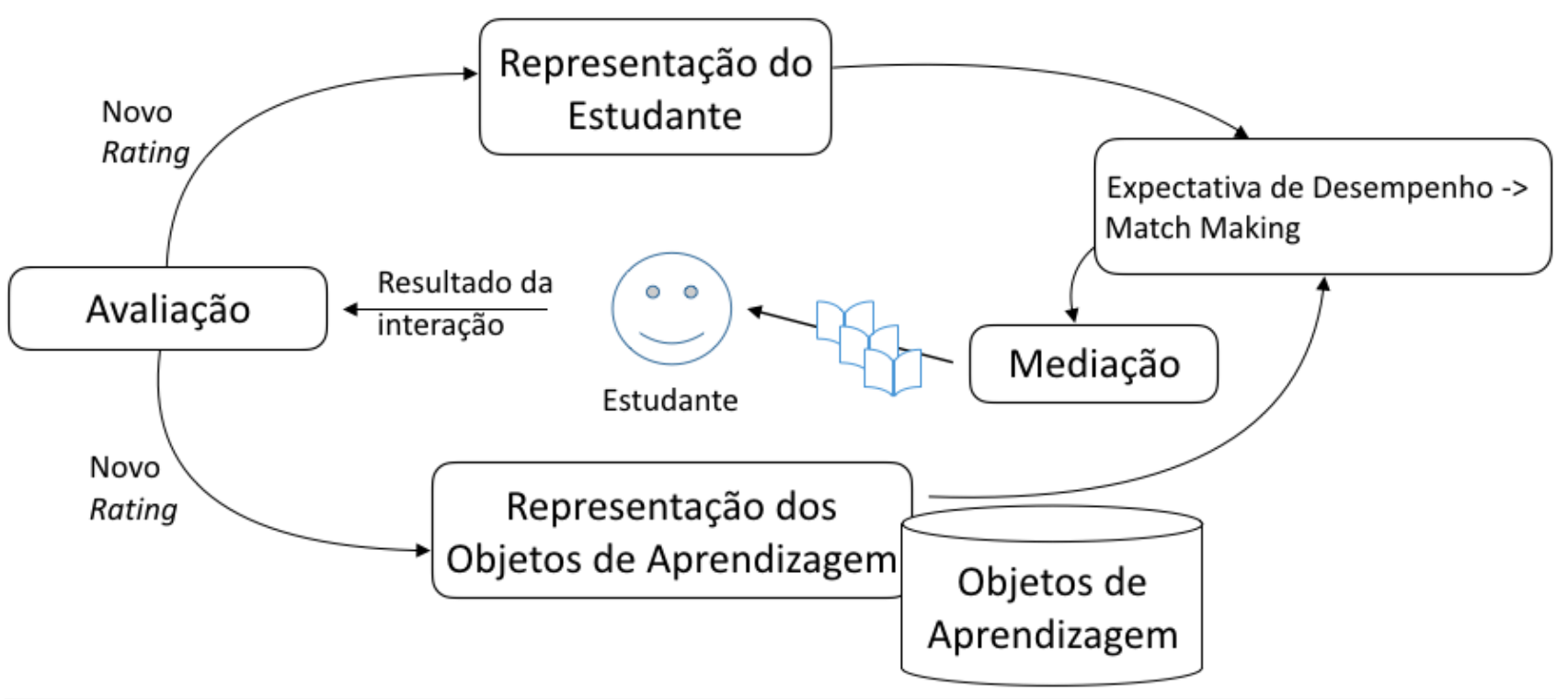

Figura 1. Arquitetura do Modelo. A avaliação se dá por um sistema de rating enquanto a mediação é feita com base em expectativa de desempenho. As interações do estudante atualizam dinamicamente o modelo.

resultado de cada interação reavalia tanto o estudante quanto o objetos de aprendizagem envolvido, atualizando seus valores de ELO.

Os estudantes passaram por uma primeira avaliação para atribuir um ELO inicial. Adotamos o TRI informado pelo ENEM como calibrador deste ELO inicial, arbitramos um ELO = 1000 para os estudantes com valor médio no TRI do ENEM. Os problemas foram avaliados por especialistas em fácil $(\mathrm{ELO}=800)$, médio $(\mathrm{ELO}=1000)$ e difícil $(\mathrm{ELO}$ $=1200)$.

A avaliação também é feita de uma maneira global, a fim de verificarmos se está havendo aprendizagem e se a recomendação foi bem sucedida. Esta avaliação é necessária como critério de parada, quando o estudante não precisa mais receber novos objetos. Uma avaliação global também serve para dar ao professor (e o próprio estudante) um relato de como está a trajetória de cada estudante. Para o caso do experimento, utilizamos estes dados para avaliar a qualidade do modelo de recomendação. Erros e acertos eventuais não são suficientes para indicar como um estudante está interagindo. Portanto, devemos nos focar no quadro maior. Podemos relacionar os comportamentos ao longo da trajetória com os perfis de interação abordados na seção 2 .

Para cada estudante, utilizamos regressão linear para avaliar a correlação entre o número da submissão e o ELO, conforme exemplificado na Figura 2. Utilizamos o coeficiente angular da reta obtida como indicador de crescimento de ELO e o erro quadrático como indicador de estabilidade de ELO. Quando o crescimento é efetivo, temos um coeficiente alto e um erro baixo. Da mesma forma, podemos avaliar períodos de assimilação (quando há uma estabilização do ELO) ou de desequilíbrio, quando o coeficiente é negativo. 


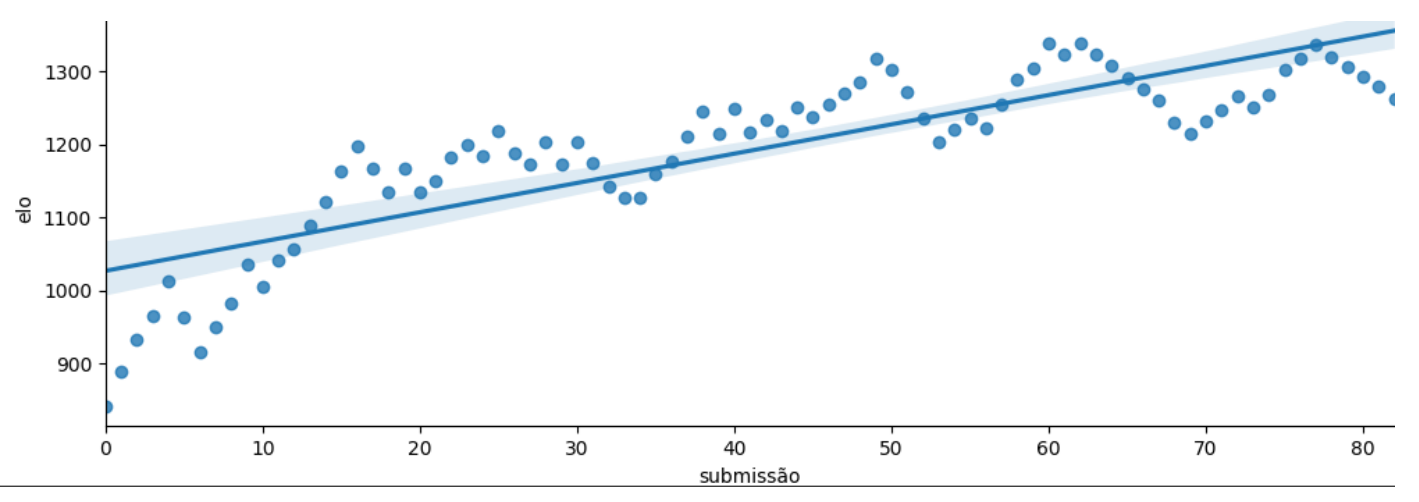

Figura 2. Representação histórica de um estudante utilizando o rating ELO. A regressão linear indica que houve um crescimento efetivo ao longo das interações.

\subsection{Mediação}

O Módulo de mediação escolhe os objetos de aprendizagem, de maneira personalizada, a partir das representações dos estudantes e objetos. O potencial de desequilíbrio é representado por $\Delta_{s, o}=R_{i-1}^{o}-R_{i-1}^{s}$, onde $i-1$ representa o instante anterior a interação. Se o $\Delta$ for muito alto, o potencial de reequilibração diminui. A medida que esta distância aumenta, mais difícil será o problema para o estudante ao ponto de este não ter as estruturas necessárias para adaptar-se ao objeto. Se o $\Delta$ for muito baixo, provavelmente o sujeito já possui os esquemas pra resolver o objeto, tornando a interação desmotivante e/ou pouco interessante para a aprendizagem. A função $s u b$ retorna uma lista de objetos $o$ adequados ao estudante $s$, tal que $P_{\min }<\Delta_{s, o}<P_{\max }$. Neste experimento, o valor padrão de $\left[P_{\min } ; P_{\max }\right]$ é $[0 ; 10]$. Esta janela pode deslocar-se (ou aumentar) conforme o comportamento do estudante.

Se o estudante obteve o mesmo resultado nas últimas três submissões, alteramos o parâmetro em função desta estabilidade. Alguns autores sugerem adaptar os desafios para evitar um estado monótono de acertos ou erros, no qual o potencial de desistência é maior [Chen et al. 2017]. O desenvolvimento de estruturas não é linear, mas opera em períodos de saltos e estabilidade [Salkind 2004]. Dessa forma, acreditamos ser importante lidar com o deslocamento da janela conforme os últimos resultados. Se o estudante obteve êxito é provável que ele esteja pronto para um "salto"em complexidade e deslocamos a janela para problemas com $\Delta$ maior. Se o estudante errou os três últimos problemas, é provável que esteja em um período de estabilidade, ou que existem conceitos que o estudante particularmente tenha mais dificuldade. Nesse caso, a janela desloca-se para $\Delta$ menores.

Se o estudante pulou uma recomendação, movemos a janela para para $\Delta$ menores. O objetivo é evitar que um erro de calibração possa afetar o estudante em seu potencial de reequilibração.

Pode ocorrer de o sistema não encontrar problemas dentro da janela de recomendação. Neste experimento, optamos por aumentar a janela, mas sem deslocala. Operamos $P_{\max }=P_{\max }+10$ e $P_{\min }=P_{\min }-10$ repetidamente enquanto a janela estiver vazia.

Os problemas selecionados pelo $\Delta$ são sorteados para recomendação, respeitando 
IX Congresso Brasileiro de Informática na Educação (CBIE 2020)

Anais do XXXI Simpósio Brasileiro de Informática na Educação (SBIE 2020)

\begin{tabular}{l|l}
\hline Duração & $7 / 03 / 2020-14 / 04 / 2020$ \\
\hline Questões & 437 \\
\hline Material de Apoio & 111 \\
\hline Estudantes convidados & 175 \\
\hline Estudantes que acessaram o sistema & 112 \\
\hline Estudantes com ao menos uma submissão & 94 \\
\hline Estudantes com mais de 30 submissões & 67 \\
\hline Submissões & 3564 \\
\hline Recomendações & 3676 \\
\hline
\end{tabular}

Tabela 1. Números sobre o experimento

a priorização de categorias. Para gerar uma tendência de balanceamento entre as categorias, os problemas de categorias menos visitadas são colocados em prioridade. Se o estudante pulou um problema recomendado, esta categoria perde prioridade na próxima recomendação.

Podemos observar o $\Delta$ como uma medida de certeza do resultado da interação. Quando há um grau alto de certeza do resultado, o modelo indica que este não é um bom caso a ser recomendado. Se temos segurança de que o estudante irá resolver o problema sem maiores desafios ou se temos segurança de que ele não irá resolver apesar de seus esforços, o problema não é boa recomendação. Quando há incerteza quanto ao resultado, o potencial de conflito cognitivo aumenta e a aprendizagem torna-se mais provável.

\section{Resultados}

A Tabela 1 apresenta alguns números acerca do experimento. Este foi feito entre março e abril de 2020. Foram convidados a participar os estudantes de cálculo dos cursos de sistemas de informação, engenharia de computação e engenharia de automação. Um total de 175 estudantes.

Foi explicado aos estudantes que a participação seria voluntária mas que os participantes ganhariam horas complementares e estariam melhor preparados em uma difícil disciplina que começaria nas próximas semanas. Enfatizamos que o desempenho no sistema não seria avaliado, apenas a participação. Dos estudantes convidados, 112 acessaram a ferramenta começando o experimento e 94 interagiram com pelo menos uma recomendação.

Ao final do processo, contamos com 3564 interações estudantes-objetos de aprendizagem. Logo que o experimento começou, a universidade entrou em recesso a fim de atender ao isolamento social. Para manter contato com os estudantes, criamos dois canais: um grupo no whatsapp e um perfil no Instagram. Através destes canais, pudemos atender os estudantes tirando suas dúvidas de como acessar o sistema. Os estudantes também retornavam para o grupo suas dificuldades, a existência de alguma questão com erro de gabarito e etc. Nos últimos dias do experimento (e seguindo por mais uma semana) os professores postaram no Instagram pequenos vídeos resolvendo os problemas com maior ELO. O uso destes canais foi limitado a fim de não criar um viés ao experimento, a fim de observar apenas o efeito do modelo.

Ao final do processo selecionamos para análise do comportamento os estudantes 


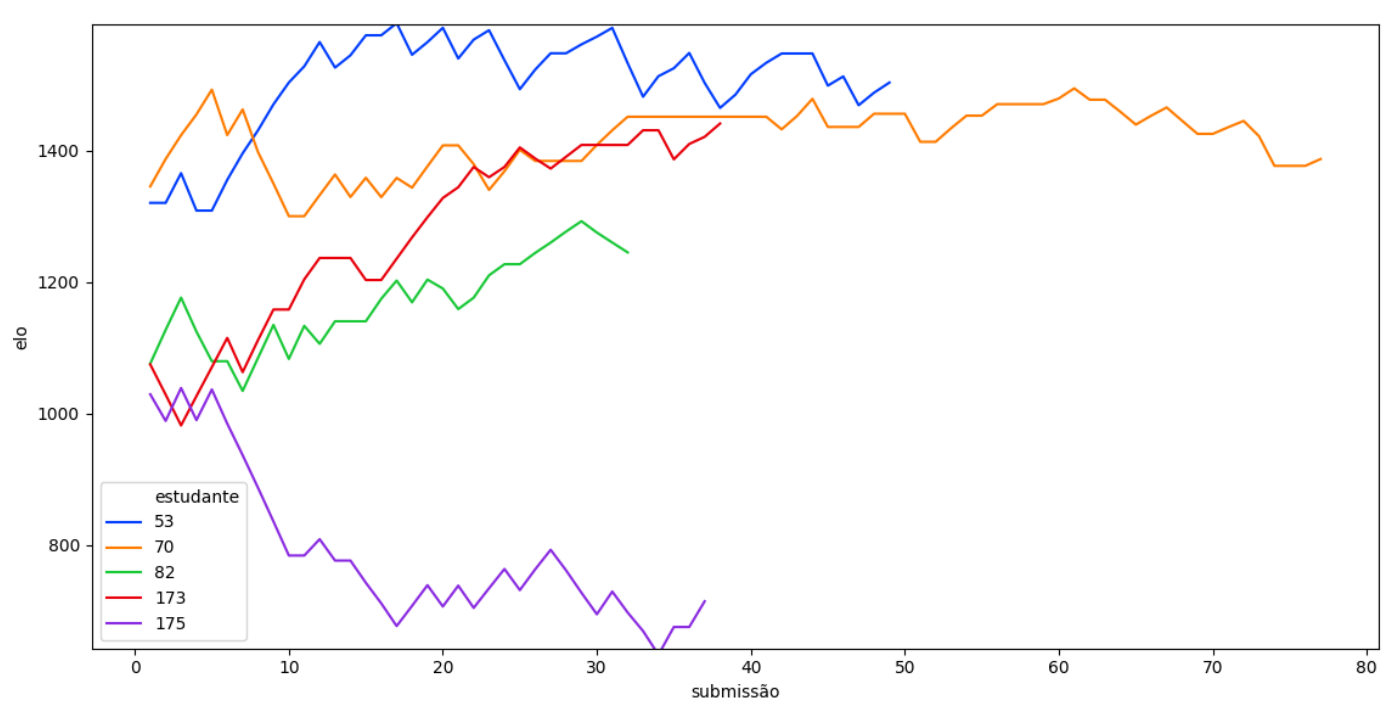

Figura 3. Representação histórica de cinco estudantes utilizando o rating ELO. Os estudantes 82 e 173 possuem trajetória indicando crescimento consistente (em diferentes ritmos). 0 estudante 70 cresce mantem-se estável (em assimilação) num ELO alto e o estudante 175 tem um decrescimento indicando que o estudante não interagiu com as recomendações como esperado. $O$ estudante 53 chega ao topo e seu comportamento indica que pode parar de interagir com o sistema.

que interagiram com os objetos como um processo, como uma "lista de exercícios" no estilo das disciplinas da engenharia, mas feita de modo personalizado e atendendo às necessidades do estudante. Consideramos para análise os estudantes com pelo menos 30 interações (submissão ou pulo). O ELO dos estudantes com menos interações ainda não está calibrado o suficiente para análise estatística. Excluímos da análise estudantes que responderam todas as questões no último dia do experimento, levando menos de $5 \mathrm{~min}$ para cada questão (entre receber a recomendação e submeter sua resposta). Este comportamento, independente do desempenho, indicou interação aleatória ou "cola". A análise final observou o comportamento de 39 estudantes. Conforme abordamos na seção 3, utilizamos o panorama geral do estudante para indicar como foi seu processo de interação com o sistema.

Observe os seguintes exemplos, apresentados na Figura 3). O estudante 173 passou por uma reequilibração majorante na sua trajetória. A medida que ele interage e acerta os problemas, ele pode interagir com problemas cada vez mais complexos. Eventualmente, ele erra algumas questões, mas isso não impede de ter um crescimento consistente. O estudante 82 também tem um crescimento, embora mais moderado. Sua taxa de acerto é menor, mas ele também consegue interagir com sucesso ao longo trajetória.

O estudante 70 não parece ter o mesmo crescimento. Este estudante pulou muitas questões (cerca de $25 \%$ ) e acaba acertando principalmente questões com $\Delta$ muito baixo. Dessa forma, ele permanece numa zona de assimilação, parecendo lidar apenas com as estruturas que já possuía. Como consequência, seu ELO tem pouco crescimento. Estudantes nesse perfil classificamos como um comportamento de assimilação. Observe como ele começa e permanece com um ELO alto e é o estudante com mais interações no gráfico (submissões e pulos). 


\begin{tabular}{l|l}
\hline Comportamento & Estudantes \\
\hline Crescimento & 30 \\
\hline Estabilização & 7 \\
\hline Decrescimento & 2 \\
\hline
\end{tabular}

Tabela 2. Classificação dos estudantes analisados

O estudante 175 (Figura 3, em vermelho) tem um comportamento que não desejamos. Ele começa com um ELO médio e vai errando até estabilizar num valor baixo. Aparentemente as recomendações não foram efetivas para ele. Nossa base, infelizmente, tinha uma amplitude limitada de problemas e talvez necessitássemos de mais problemas básicos. Estudantes nesse perfil classificamos como "reequilibração não majorante", de decrescimento.

O estudante 53 chega ao topo rapidamente. Durante o experimento, haviam poucas questões com elo acima de 1400. Quando ele chega perto de 1600, começa a estabilizar. Embora sua taxa de acerto seja alta, seus acertos geram pouco crescimento de ELO enquanto eventuais erros geram punições fortes. Nesse caso, avaliamos que o estudante já atingiu o nível necessário. Fora do experimento, o nosso mediador usaria padrão como critério de parada.

Através da análise da variação do ELO ao longo das interações, os estudantes foram classificados conforme apresentados na Tabela 1. 30 deles teve crescimento consistente de ELO, resolvendo problemas cada vez mais complexos 2 deles teve uma queda em relação ao seu ELO inicial, mesmo que aparentemente estes levassem tempo considerável para resolver as questões e consultassem material de apoio. As recomendações não foram bem feitas para eles ou fatos externos ao experimento atrapalharam a experiência dos estudantes. 7 estudantes tiveram crescimento e estabilização. 2 por pulo (semelhante a estudante 70), 5 por estabilização (por topo ou por falta de problemas na zona de recomendação. ${ }^{2}$

Os resultados foram promissores e indicam que o modelo ELO para personalização de sistemas de recomendação é uma boa alternativa em sistemas online no ensino híbrido. Os estudantes relataram ter gostado da ferramenta e da integração com WhatsApp e Instagram. Eles apontaram que foi uma iniciativa que os ajudou a manter contato com a universidade mesmo em isolamento. Os professores especialistas começaram o processo de inserir questões de cálculo e GAAL ${ }^{3}$ e seguir com os estudantes durante as aulas a distância. Além dos estudantes que participaram do experimento, estudantes de outros cursos serão adicionados nesse processo.

\section{Referências}

Bacich, L., Neto, A. T., and de Mello Trevisani, F. (2015). Ensino híbrido: personalização e tecnologia na educação. Penso Editora.

\footnotetext{
${ }^{2} \mathrm{Em}$ alguns casos, quando os estudantes atingiam o ELO de cerca de 1450, pouco (ou nenhum) problema estava disponível a ele nesta zona de recomendação. O sistema, como apresentado na arquitetura, aumentava a zona de recomendação dando eventualmente apenas problemas de ELO muito baixo. O estudante, apesar de ter boa taxa de acerto, tinha um crescimento pequeno. A medida que o ELO das questões mais difíceis crescia, este problema foi diminuindo.

${ }^{3}$ Geometria Analítica e Álgebra Linear
} 
IX Congresso Brasileiro de Informática na Educação (CBIE 2020)

Anais do XXXI Simpósio Brasileiro de Informática na Educação (SBIE 2020)

Becker, F. (2008). Modelos pedagógicos e modelos epistemológicos. Metodologia: construção de uma proposta científica. Curitiba: Camões, pages 45-56.

BRASIL, M. (2009). Matriz de referência para o enem 2009. Brasília:[sn].

BRASIL, M. (2017). Base nacional comum curricular. Brasilia DF MEC Secretaria de Educacao Basica.

Chen, Z., Xue, S., Kolen, J., Aghdaie, N., Zaman, K. A., Sun, Y., and Seif El-Nasr, M. (2017). Eomm: An engagement optimized matchmaking framework. In Proceedings of the 26th International Conference on World Wide Web, pages 1143-1150. International World Wide Web Conferences Steering Committee.

da SILVA, J. A. (2011). Repetição e desafio nos exercícios escolares: dois lados de uma mesma moeda. Schème-Revista Eletrônica de Psicologia e Epistemologia Genéticas, 1(1):95-107.

Elo, A. E. (1978). The rating of chessplayers, past and present. Arco Pub.

Knight, C. C. and Sutton, R. E. (2004). Neo-piagetian theory and research: enhancing pedagogical practice for educators of adults. London Review of Education, 2(1):47-60.

Langville, A. N. and Meyer, C. D. (2012). Who's\# 1?: the science of rating and ranking. Princeton University Press.

Lefrançois, G. R. (2012). Theories of human learning: What the professor said. Cengage Learning.

Piaget, J. (1972). Intellectual evolution from adolescence to adulthood. Human development, 15(1):1-12.

Prisco, A., dos Santos, R., Botelho, S., Tonin, N., and Bez, J. (2017). Using information technology for personalizing the computer science teaching. In 2017 IEEE Frontiers in Education Conference (FIE), pages 1-7. IEEE.

Prisco, A., Penna, R., Botelho, S., Tonin, N., Bez, J., et al. (2018). A multidimensional elo model for matching learning objects. In 2018 IEEE Frontiers in Education Conference (FIE), pages 1-9. IEEE.

Salkind, N. J. (2004). An introduction to theories of human development. Sage Publications.

Smole, K. S. and Diniz, M. I. (2009). Ler, escrever e resolver problemas: habilidades básicas para aprender matemática. Artmed Editora.

Teixeira, C. d. J. (2019). A proposição de problemas como estratégia de aprendizagem da matemática: Uma ênfase sobre efetividade, colaboração e criatividade.

Vargas, A. P., dos Santos, R. A. P., Bez, J., Tonin, N., and da Costa Botelho, S. S. (2019). Um modelo de mediação pedagógica para ambientes massivos. RENOTE-Revista Novas Tecnologias na Educação, 17(1):93-102.

Zaffalon, F., Vargas, A. P., de Souza, R. L., Penna, R., Bez, J., Tonin, N., and da Costa Botelho, S. S. (2019). Um estudo comparativo entre dois modelos que estimam a habilidade dos estudantes: Elo e teoria de resposta ao item. In Brazilian Symposium on Computers in Education (Simpósio Brasileiro de Informática na Educação-SBIE), volume 30, page 459. 